\title{
Market Share Delegation in a Bertrand Duopoly: Synchronisation and Multistability
}

\author{
Luciano Fanti, ${ }^{1}$ Luca Gori, ${ }^{2}$ Cristiana Mammana, ${ }^{3}$ and Elisabetta Michetti ${ }^{3}$ \\ ${ }^{1}$ Department of Economics and Management, University of Pisa, Via Cosimo Ridolfi 10, 56124 Pisa, Italy \\ ${ }^{2}$ Department of Law, University of Genoa, Via Balbi 30/19, 16126 Genoa, Italy \\ ${ }^{3}$ Department of Economics and Law, University of Macerata, Via Crescimbeni 20, 62100 Macerata, Italy
}

Correspondence should be addressed to Elisabetta Michetti; elisabetta.michetti@unimc.it

Received 21 October 2014; Revised 2 March 2015; Accepted 9 March 2015

Academic Editor: Jinde Cao

Copyright (C) 2015 Luciano Fanti et al. This is an open access article distributed under the Creative Commons Attribution License, which permits unrestricted use, distribution, and reproduction in any medium, provided the original work is properly cited.

\begin{abstract}
This paper tackles the issue of local and global analyses of a duopoly game with price competition and market share delegation. The dynamics of the economy is characterised by a differentiable two-dimensional discrete time system. The paper stresses the importance of complementarity between products as a source of synchronisation in the long term, in contrast to the case of their substitutability. This means that when products are complements, players may coordinate their behaviour even if initial conditions are different. In addition, there exist multiple attractors so that even starting with similar conditions may end up generating very different dynamic patterns.
\end{abstract}

\section{Introduction}

Strategic delegation is a relevant topic in both oligopoly theory and industrial organisation, and several papers have contributed to clarify questions related to the differences between the behaviour of profit-maximising firms and managerial firms (e.g., [1-4]). In the former kind of firms, ownership and management coincide, and consequently the main aim they pursue is profit maximisation. In the latter, ownership and management are separate and managers may be driven by incentive schemes that only partially take into account profit and the other objectives of the firms, such as output, revenues, relative performance evaluation, and market share $[3,5-10]$. In addition to the above-mentioned theoretical papers, there also exist some empirical works that stress the importance of market share delegation contracts in actual economies [11, 12].

The present paper studies a nonlinear duopoly game with price competition and market share delegation and extends the study carried out by Fanti et al. [13] to the case of complementary or independent products. To this end, by following an established literature led by Bischi et al. [14], we assume that players have limited information and analyse how a managerial incentive scheme based on market share affects the local and global dynamics of a two-dimensional discrete time system. The paper stresses the differences with the analysis carried out by Fanti et al. [13] on the substitutability between products in the case with managerial firms and market share contracts and compares the results achieved.

The rest of the paper is organised as follows. Section 2 describes the model. Section 3 shows some preliminary global properties of the two-dimensional dynamic system (feasible set). Section 4 studies the fixed points of the system, the invariant sets, and local stability. Section 5 is concerned with multistability and shows that synchronisation may arise when managers receive the same bonus. It also stresses the differences with Fanti et al. [13] and takes into account the asymmetric case in which bonuses are not equally weighted in the managers' objective function. Section 6 outlines the conclusions.

\section{The Model}

Consider a duopoly game with price competition, horizontal differentiation, and market share delegation contracts 
(see [13] for details). Market demands of goods 1 and 2 are, respectively, given by

$$
\begin{aligned}
& q_{1}=\frac{1-p_{1}-d\left(1-p_{2}\right)}{1-d^{2}} \\
& q_{2}=\frac{1-p_{2}-d\left(1-p_{1}\right)}{1-d^{2}}
\end{aligned}
$$

where $d \in(-1,0]$ is the degree of differentiation of (complementary) products, while $q_{i} \geq 0$ and $p_{i} \geq 0$ are quantity and price per unit of good of firm $i(i=1,2)$.

Both the firms have the same marginal cost $0 \leq w<1$ and hire a manager, who receives a bonus based on market share $q_{i} /\left(q_{i}+q_{j}\right)(i, j=1,2, i \neq j)$, where $q_{i}+q_{j}$ is total supply. The objective function of manager $i$ is

$$
W_{i}=\Pi_{i}+b_{i} \frac{q_{i}}{q_{i}+q_{j}}, \quad i, j=1,2, i \neq j,
$$

where $\Pi_{i}=\left(p_{i}-w\right) q_{i}$ are profits and $b_{i}>0$ is the (constant) delegation variable of player $i$. Hence, by using (1), (2) becomes

$$
\begin{array}{r}
W_{i}=\frac{1-p_{i}-d\left(1-p_{j}\right)}{1-d^{2}}\left[p_{i}-w+b_{i} \frac{1+d}{2-p_{i}-p_{j}}\right], \\
i, j=1,2, \quad i \neq j,
\end{array}
$$

from which we get the following marginal bonus:

$$
\begin{array}{r}
\frac{\partial W_{i}}{\partial p_{i}}=\frac{1-2 p_{i}-d\left(1-p_{j}\right)+w}{1-d^{2}}-b_{i} \frac{(1+d)\left(1-p_{j}\right)}{(1-d)\left(2-p_{i}-p_{j}\right)^{2}}, \\
i, j=1,2, \quad i \neq j .
\end{array}
$$

We now assume a discrete time $\left(t \in \mathbb{Z}_{+}\right)$dynamic setting, where each player $i$ has limited information, as in Bischi et al. [14], and uses the following behavioural rule to set the price for the subsequent period:

$$
p_{i, t+1}=p_{i, t}+\alpha p_{i, t} \frac{\partial W_{i}\left(p_{i, t}, p_{j, t}\right)}{\partial p_{i, t}}, \quad i=1,2, t \in \mathbb{Z}_{+},
$$

where $\alpha>0$. We want to describe the qualitative and quantitative long-term price dynamics when products are complementary or independent, that is, $d \in(-1,0]$, and underline the similarities and differences with the case of substitutability investigated in Fanti et al. [13].
Assume $x^{\prime}=p_{1, t+1}, x=p_{1, t}, y^{\prime}=p_{2, t+1}$, and $y=p_{2, t}$. By using (4) and (5), the two-dimensional discrete time dynamic system is as follows:

$$
T:\left\{\begin{aligned}
x^{\prime} & =x F(x, y) \\
& =x\left[1+\alpha\left(\frac{1-2 x-d(1-y)+w}{1-d^{2}}\right.\right. \\
y^{\prime} & =y G(x, y) \\
& =y\left[1+\alpha\left(\frac{1-2 y-d(1-x)+w}{(1-d)(2-x-y)^{2}}\right)\right] \\
& \left.\left.-b_{2} \frac{(1+d)(1-x)}{(1-d)(2-x-y)^{2}}\right)\right] .
\end{aligned}\right.
$$

\section{The Feasible Set}

It is of importance to observe that system (6) is economically meaningful only whether, at any time $t$, the two state variables $x$ and $y$ are not negative; that is, they belong to $Q$, where $Q$ is the convex polygon with vertices $(0,0),(0,1-d),(1,1)$, and $(1-d, 0)$.

Let $T^{t}(x(0), y(0)), t=0,1,2 \ldots$, denote the $t$ th iterate of system $T$ for a given initial condition $(x(0), y(0)) \in Q$. Then, the sequence $\psi_{t}=\{(x(t), y(t))\}_{t=0}^{\infty}$ is called trajectory. A trajectory $\psi_{t}$ is said to be feasible for $T$ if $(x(t), y(t)) \in Q$ for all $t \in \mathbb{N}$; otherwise, it is unfeasible. The set $D \subseteq Q$ whose points generate feasible trajectories is called feasible set. A point belonging to the feasible set is called feasible point.

The feasible set of system $T$ is depicted in white in Figures 1(a) and 1(b) for two different parameter constellations, while the unfeasible points belonging to $Q$ are depicted in grey. The following evidence can be immediately observed: similarly to the substitutability case, (i) set $D$ is nonempty such that $D \subset Q$ and (ii) set $D$ may have a simple structure (as in Figure 1(a)) or a complex structure (as in Figure 1(b)). The first evidence can be easily demonstrated by considering that the origin is a feasible point and that there exists a $\eta>0$ small enough such that $(1-\eta, 1-\eta)$ is not a feasible point.

With regard to the study of the structure of the feasible set, a numerical procedure based on the study of the properties of the critical curves can be used (see, e.g., [15-17]). By taking into account the results proved in Fanti et al. [13], it is easy to verify that $T$ is of $Z_{4}-Z_{2}-Z_{0}$ type since $Q$ can be subdivided into regions whose points have 4,2 , or 0 preimages, and the boundaries of such regions are characterised by the existence of at least two coincident preimages.

We denote the critical curve of rank-1 by LC (it represents the locus of points that have two or more coincident preimages) and the curve of merging preimages by $\mathrm{LC}_{-1}$; that is, the set of points $(x, y) \in Q$ such that $|J(x, y)|=0$, where 


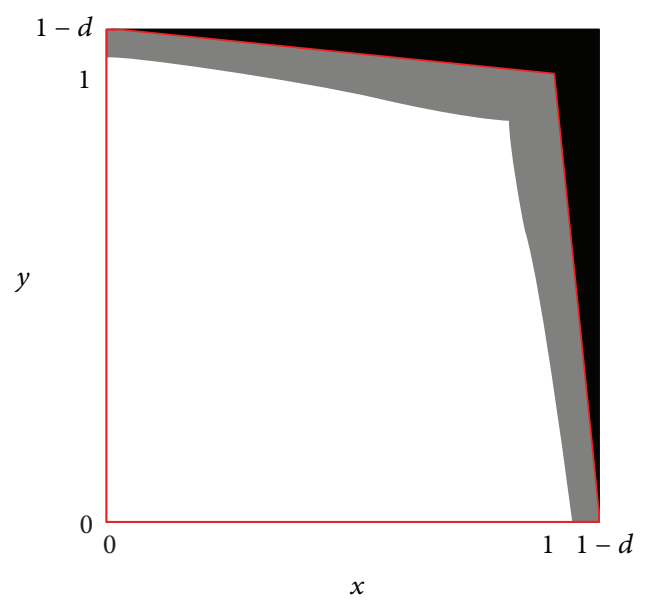

(a)

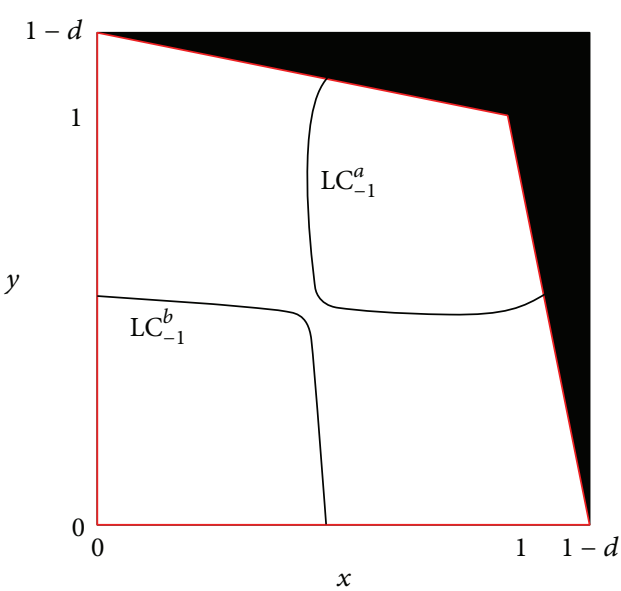

(c)

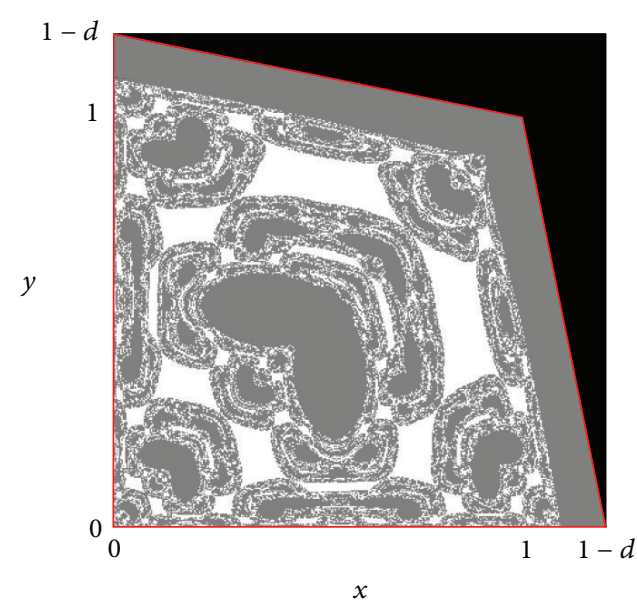

(b)



(d)

Figure 1: (a), (b). The feasible set $D \subset Q$ is depicted in white; the gray points are initial conditions producing unfeasible trajectories. (a) $\alpha=1.5, d=-0.1, w=0.5$, and $b_{1}=b_{2}=0.2$. (b) $\alpha=1.5, d=-0.2, w=0.5$, and $b_{1}=b_{2}=0.2$. (c) Critical curves of rank- 0 , LC L $_{-1}$, for system $T$ and the parameter values as in (b). (d) Critical curves of rank-1, LC $=T\left(\mathrm{LC}_{-1}\right)$, for the same parameter values as in panel (c). These curves separate the plane into the regions $Z_{4}, Z_{2}$, and $Z_{0}$, whose points have a different number of preimages.

$$
J(x, y)=\left(\begin{array}{cc}
1+\alpha\left(\frac{1-4 x-d(1-y)+w}{1-d^{2}}-\frac{b_{1}(1+d)(1-y)(2+x-y)}{(1-d)(2-x-y)^{3}}\right) & \alpha x\left(\frac{d}{1-d^{2}}-b_{1} \frac{(1+d)(x-y)}{(1-d)(2-x-y)^{3}}\right) \\
\alpha y\left(\frac{d}{1-d^{2}}-b_{2} \frac{(1+d)(y-x)}{(1-d)(2-x-y)^{3}}\right) & 1+\alpha\left(\frac{1-4 y-d(1-x)+w}{1-d^{2}}-\frac{b_{2}(1+d)(1-x)(2-x+y)}{(1-d)(2-x-y)^{3}}\right)
\end{array}\right)
$$

is the Jacobian matrix of system $T$ (see Figures $1(\mathrm{c})$ and $1(\mathrm{~d})$ ).

The study of the structure of set $D$ is of interest from both economic and mathematical perspectives, since the long-term evolution of the economic system becomes pathdependent, and a thorough knowledge of the properties of $D$ becomes crucial in order to predict the system's feasibility.

We now fix all parameter values but $d$. Then, as is shown in Figures 2(a) and 2(b), if $d=-0.15$, set $D$ has a simple structure (connected set), while when $d=-0.2$, set $D$ consists of infinitely many nonconnected sets. This is due to the fact that the $\mathrm{LC}^{b}$ curve moves upwards as parameter $d$ decreases and consequently a threshold value $\bar{d} \simeq-0.1551$ does exist such that a contact between a critical curve and the boundary of the feasible set occurs. This contact bifurcation causes the change of $D$ from a connected set to a nonconnected set. In fact, a portion of the unfeasible set enters in a region characterised by a high number of preimages so that new components of the unfeasible set suddenly appear after the contact (in Figure 2(c), the feasible set is depicted immediately after the contact bifurcation creating grey holes inside the white region). The complexity of the structure of the feasible set increases if $d$ decreases and the grey area 


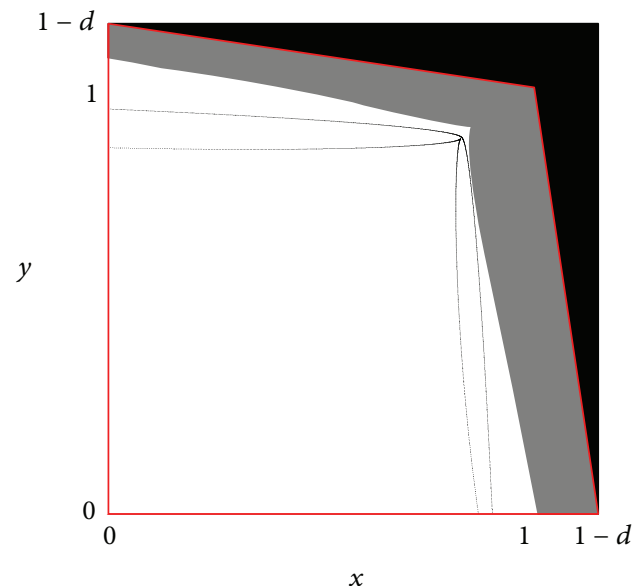

(a)

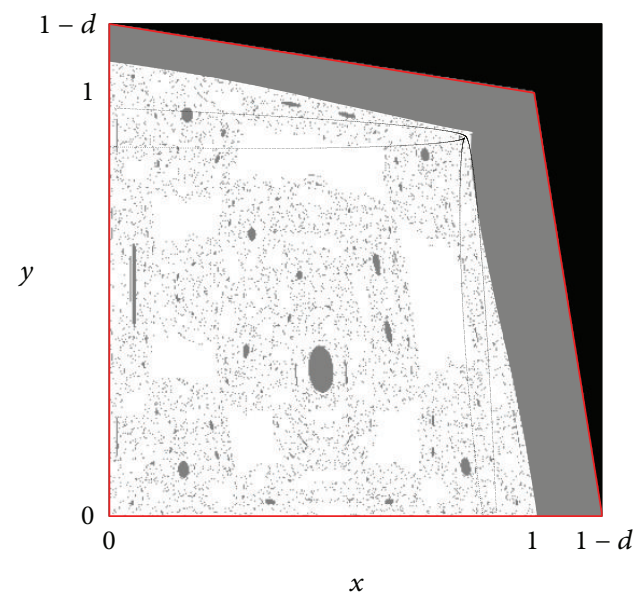

(c)

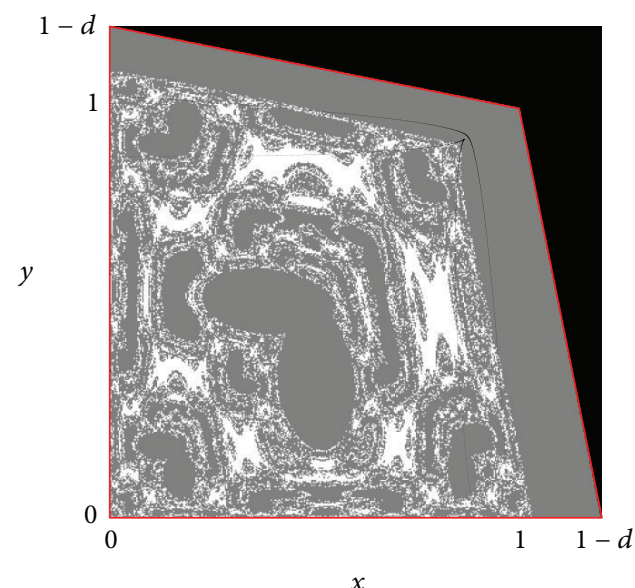

(b)

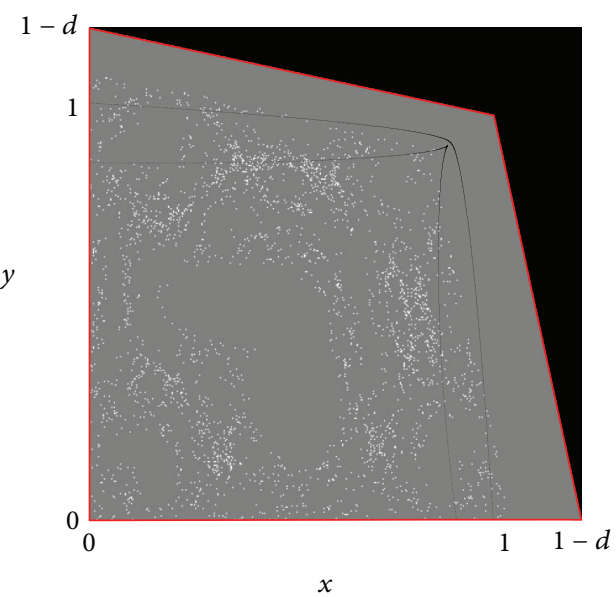

(d)

Figure 2: Parameter values: $\alpha=1.5, w=0.5, b_{1}=0.4$, and $b_{2}=0.2$. (a) $D$ has a simple structure $(d=-0.15)$. (b) $D$ has a complex structure $(d=-0.2)$. (c) Set $D$ after the contact bifurcation $(d=-0.16)$ : gray holes are depicted. (d) Immediately before the final bifurcation $(d=-0.216)$ almost all trajectories are unfeasible.

increases too (i.e., the set of initial conditions generating unfeasible trajectories); however, it can be also observed that if $d$ decreases further, a final bifurcation occurs. In fact, as $d$ crosses a value $d \simeq-0.218$, almost all trajectories become unfeasible (see Figure 2(d)).

Notice that in the simulations presented in Figure 2 we have assumed $b_{1} \neq b_{2}$. However, a similar behaviour holds also when $b_{1}=b_{2}$ : in this case, the contact bifurcation between the critical set and the boundary of the feasible set occurs at two points that are symmetric with respect to the main diagonal, so that the resulting feasible set is symmetric too (see Figures 1(a) and 1(b)). The previous arguments show that the bifurcations concerning the structure of the feasible set is strictly related to the value of the two key parameters $d$ and $b_{i}$, which represent the degree of horizontal product differentiation and the level of market share bonuses. The following results can be proved.

Proposition 1. Let $T$ be the dynamic system given by (6).

(i) If $d \rightarrow-1^{+}$, then $D=\{(0,0)\}$.

(ii) If $b_{i} \rightarrow+\infty, i=1,2$, then $D=\{(0,0)\}$.
Proof. (i) If $d \rightarrow-1^{+}$, then $Q \rightarrow I=\left\{(x, y) \in \mathbb{R}_{+}^{2}: x+y \leq\right.$ $2, x \geq 0, y \geq 0\}$. Observe first that it must be $x+y<2$ for $T$ being well defined and that $(0,0) \in D$, and hence we consider initial conditions such that $x(0)+y(0)<2$ and at least one component of $(x(0), y(0))$ is strictly positive. Taking into account system (6), it can be observed that

$$
x(1)+y(1)=x(0)+y(0)+\omega(d, x(0), y(0))
$$

and that $\forall(x(0), y(0)) \in Q: x(0)+y(0)<2$ and $x(0)+$ $y(0) \neq 0$; if $d \rightarrow-1^{+}$, then $\omega(d, x(0), y(0))$ diverges; that is, $(x(0), y(0))$ produces an unfeasible trajectory.

(ii) This statement can be proved simply considering the limits $\lim _{b_{1} \rightarrow+\infty} x(1)$ and $\lim _{b_{2} \rightarrow+\infty} y(1)$ for any given initial point $(x(0), y(0)) \in Q, x(0)+y(0) \neq 0$.

According to Proposition 1 , if $b_{i}$ is high enough or products tend to be complements ( $d$ is low enough), the feasible set is consists of only the origin. This result confirms the one obtained in Fanti et al. [13] for the substitutability case; that is, economic meaningful dynamics are produced only when the degree of complementarity or substitutability 
between products is not too high. More precisely, in the case under investigation, economically meaningful longterm dynamics can be produced only for $d \in I_{-}(0)$ and $b_{i} \epsilon$ $I_{+}(0)(i=1,2)$, thus confirming the numerical experiments previously presented.

By taking into account the above-mentioned arguments, in what follows we will focus on the study of the dynamics produced by $T$ by assuming that $b_{i}$ is sufficiently small and $d$ is sufficiently high.

\section{Fixed Points, Invariant Sets, and Local Stability}

Let $T$ be the dynamic system given by (6) and consider a feasible initial condition. We now recall the results proved in Fanti et al. [13] concerning fixed points and other invariant sets of $T$ for $d \in(0,1)$. It can be verified that they still hold also when $d \in(-1,0]$. We here present a sketch of the proof and we refer to Fanti et al. [13] for a further discussion.

Remark 2. Let $T$ be given by (6). Then, one has the following.

(i) $I_{x}=\{(x, y): 0 \leq x \leq 1-d, y=0\}$ and $I_{y}=\{(x, y)$ : $0 \leq y \leq 1-d, x=0\}$ are invariant sets. The dynamics of $T$ on such sets are governed by $\phi_{x}=x F(x, 0)$ and $\phi_{y}=y G(0, y)$ and they can be complex; in any case, $I_{x}$ and $I_{y}$ are repellor.

(ii) If $b_{1}=b_{2}=b$, then also $\Delta=\left\{(x, y) \in \mathbb{R}_{+}^{2}: x=\right.$ $y, x \in[0,1)\}$ is an invariant set. The dynamics of $T$ on such a set are governed by $\phi(x)=x[1+(\alpha /(1-d))$ $(((1-d)(1-x)-x+w) /(1+d)-b((1+d) / 4(1-x)))]$ and they can be complex; furthermore, $\Delta$ can be an attracting set.

(iii) The origin $E_{0}=(0,0)$ is a fixed point for all parameter values; it can be a stable node, an unstable node, or a saddle point. Up to two more fixed points on $I_{y}$ and $I_{x}$ may be owned. They are given by $E_{1}=\left(0, y_{0}\right)$ and $E_{2}=\left(x_{0}, 0\right)$ and can be unstable nodes or saddle points.

(iv) If $b_{1}=b_{2}=b$, then $T$ admits a unique interior fixed point $E_{b}^{*}=\left(x_{b}^{*}, x_{b}^{*}\right)$ for all $b<4(1-d+w) /$ $(1+d)^{2}=\bar{b}$, where $x_{b}^{*}=1-((1-w)+$ $\left.\sqrt{(1-w)^{2}+b(2-d)(1+d)^{2}}\right) / 2(2-d) . E_{b}^{*}$ can be a stable node, an unstable node, or a saddle point.

Proof. Consider the system $T$ given by (6).

(i) $T(x, 0)=\left(x^{\prime}, 0\right)$ and $T(0, y)=\left(0, y^{\prime}\right)$; that is, $I_{x}$ and $I_{y}$ are invariant, and consequently the dynamics of $T$ on such lines are governed by the two one-dimensional maps $\phi_{x}=x F(x, 0)$ and $\phi_{y}=y G(0, y)$. As both $\phi_{x}$ and $\phi_{y}$ are unimodal for suitable parameter values (as proved in [13]), complex dynamics can be produced. $I_{x}$ and $I_{y}$ are repellor as the eigenvalue of $J(x, 0)$ and $J(0, y)$ associated with the direction orthogonal to each semiaxis is greater than one for all parameter values. (ii) Assume $b_{1}=b_{2}=b$; then, $T(x, x)=\left(x^{\prime}, x^{\prime}\right)$, and hence $\Delta$ is invariant and the dynamics of system $T$ on $\Delta$ are governed by $\phi(x)=x F(x, x)$. Given the properties of $\phi(x)$ studied in Fanti et al. [13], nonmonotonicity can occur for suitable parameter values and complex dynamics may emerge. Finally, $\Delta$ can be an attracting set as the eigenvalue of $J(x, x)$ associated with the eigenvector orthogonal to the diagonal may be less than one in modulus (see again Fanti et al. [13]).

(iii) Since $T(0,0)=(0,0), E_{0}$ is a fixed point; furthermore, while considering $J(0,0)$, it can be observed that $E_{0}$ can be a stable node, an unstable node, or a saddle point. By solving $\phi_{x}=x$ and $\phi_{y}=y$ and following Fanti et al. [13], it can be easily verified that up to two positive solutions exist, namely, $E_{1}$ and $E_{2}$; such fixed points cannot be stable nodes as $I_{x}$ and $I_{y}$ are repellor.

(iv) Assume $b_{1}=b_{2}=b$; then, by solving equation $\phi(x)=$ $x$ it can be verified that it admits a unique positive feasible solution $x_{b}^{*}$ iff $b<\bar{b}$ (see Fanti et al. [13] for more details); as each eigenvalue of $J\left(x_{b}^{*}, x_{b}^{*}\right)$ can be greater or less than one in modulus, then $E_{b}^{*}$ can be a stable node, an unstable node, or a saddle point.

The question of the existence of an interior fixed point in the general case $b_{1} \neq b_{2}$ (i.e., with different market share bonuses) cannot be addressed analytically. However, it will be discussed later in the paper by using numerical techniques. By taking into account parts (i) and (iii) in Remark 2, in what follows we will focus on the study of the dynamics produced by $T$ for a feasible initial condition belonging to the interior of $Q$, thus focusing on economically meaningful initial states.

\section{Synchronisation and Multistability}

In order to study the evolution of system $T$ when products are independent or complementary and compare this case with that of substitutable goods, we concentrate on the particular case of identical market share bonuses; that is, $b_{1}=b_{2}=b$. As a consequence, system $T$ in (6) takes the symmetric form $T_{b}$ given by

$$
T_{b}:\left\{\begin{aligned}
& x^{\prime}=x f(x, y) \\
&=x\left[1+\alpha\left(\frac{1-2 x-d(1-y)+w}{1-d^{2}}\right.\right. \\
&\left.\left.-b \frac{(1+d)(1-y)}{(1-d)(2-x-y)^{2}}\right)\right] \\
& y^{\prime}=y g(x, y) \\
&=y\left[1+\alpha\left(\frac{1-2 y-d(1-x)+w}{1-d^{2}}\right)\right. \\
&\left.\left.-b \frac{(1+d)(1-x)}{(1-d)(2-x-y)^{2}}\right)\right] .
\end{aligned}\right.
$$


Since map $T_{b}$ is symmetric, that is, it remains the same when the players are exchanged, then either an invariant set of the map is symmetric with respect to $\Delta$ or its symmetric set is invariant. By considering part (iv) in Remark 2, the local stability analysis of the unique interior fixed point $E_{b}^{*}$ can be carried out by considering the Jacobian matrix associated with system $T_{b}$ given by

$$
J_{b}(x, y)=\left(\begin{array}{cc}
1+\alpha\left(\frac{1-4 x-d(1-y)+w}{1-d^{2}}-\frac{b(1+d)(1-y)(2+x-y)}{(1-d)(2-x-y)^{3}}\right) & \alpha x\left(\frac{d}{\left.1-d^{2}-b \frac{(1+d)(x-y)}{(1-d)(2-x-y)^{3}}\right)}\right. \\
\alpha y\left(\frac{d}{1-d^{2}}-b \frac{(1+d)(y-x)}{(1-d)(2-x-y)^{3}}\right) & 1+\alpha\left(\frac{1-4 y-d(1-x)+w}{1-d^{2}}-\frac{b(1+d)(1-x)(2-x+y)}{(1-d)(2-x-y)^{3}}\right)
\end{array}\right)
$$

Let

$$
\begin{gathered}
J_{1}(x)=1+\frac{\alpha}{1-d^{2}} \\
\cdot \frac{4(4-d)(1-x)^{3}+4(w-3)(1-x)^{2}-b(1+d)^{2}}{4(1-x)^{2}}, \\
J_{2}(x)=\frac{\alpha d}{1-d^{2}} x .
\end{gathered}
$$

Then, the Jacobian matrix evaluated at a point on the diagonal $\Delta$ is of the kind

$$
J_{b}(x, x)=\left(\begin{array}{ll}
J_{1}(x) & J_{2}(x) \\
J_{2}(x) & J_{1}(x)
\end{array}\right),
$$

so that the eigenvalues of $J_{b}(x, x)$ are both real and given by

$$
\begin{aligned}
& \lambda_{b \|}(x)=J_{1}(x)+J_{2}(x), \\
& \lambda_{b \perp}(x)=J_{1}(x)-J_{2}(x),
\end{aligned}
$$

while the corresponding eigenvectors are, respectively, given by $\underline{v}_{b \|}=(1,1)$ and $\underline{v}_{b \perp}=(1,-1)$.

The eigenvalues evaluated at the fixed point $E_{b}^{*}$ are, respectively,

$$
\begin{aligned}
& \lambda_{b \|}\left(E_{b}^{*}\right)=J_{1}\left(E_{b}^{*}\right)+J_{2}\left(E_{b}^{*}\right), \\
& \lambda_{b \perp}\left(E_{b}^{*}\right)=J_{1}\left(E_{b}^{*}\right)-J_{2}\left(E_{b}^{*}\right) .
\end{aligned}
$$

Thus, $E_{b}^{*}$ can be attracting for suitable values of parameters such that both $\lambda_{\|}\left(E_{b}^{*}\right)$ and $\lambda_{\perp}\left(E_{b}^{*}\right)$ belong to the set $(-1,1)$.

Different from the case in which products are substitutes, the following Proposition can easily be verified.

Proposition 3. If $d=0$, then $\lambda_{b \|}\left(E_{b}^{*}\right)=\lambda_{b \perp}\left(E_{b}^{*}\right)$; if $d \in$ $(-1,0)$, then $\lambda_{b \|}\left(E_{b}^{*}\right)<\lambda_{b \perp}\left(E_{b}^{*}\right)$.

Proof. If $d=0$, then $J_{2}(x)=0 \forall x$, and consequently $\lambda_{b \|}\left(E_{b}^{*}\right)=\lambda_{b \perp}\left(E_{b}^{*}\right)$; if $d \in(-1,0)$, then $J_{2}(x)<0 \forall x>0$, and hence $\lambda_{b \|}\left(E_{b}^{*}\right)<\lambda_{b \perp}\left(E_{b}^{*}\right)$.

From Proposition 3 it follows that if $d=0$, then the interior fixed point is a stable or an unstable node. Furthermore, conditions $\lambda_{b \|}\left(E_{b}^{*}\right)>1$ or $\lambda_{b \perp}\left(E_{b}^{*}\right)<-1$ are sufficient for $E_{b}^{*}$ to be an unstable node.
The following condition for the local stability of $E_{b}^{*}$ (which holds if products are substitutes) applies also to the case $d \in(-1,0]$ and it can be recalled below (see Fanti et al. [13] for the proof).

Proposition 4. Let system $T_{b}$ be given by (9). Then $a \epsilon>0$ does exist such that $E_{b}^{*}$ is locally asymptotically stable $\forall b \in(\bar{b}-\epsilon, \bar{b})$, given the other parameter values.

By taking into account Propositions 3 and 4, a sufficient condition for $E_{b}^{*}$ to be locally stable for $d=0$ is $b \rightarrow 4(1+w)^{-}$. In this case, given the geometric properties of map $\phi(x)$ described in Fanti et al. [13], the initial conditions belonging to $\Delta$ converge to $x_{b}^{*}$ with independent products. In addition, the trajectories starting from initial conditions close to it, that is, $(x(0), y(0)) \in I\left(E_{b}^{*}, r\right)$, with $x(0) \neq$ $y(0)$, also converge to $E_{b}^{*}$. This behaviour occurs as long as $d \in I_{-}(0)$, while, as $d$ decreases, the fixed point loses its stability firstly along the diagonal, thus giving rise to a different scenario compared to that presented in Fanti et al. [13] for the substitutability case.

Definition 5. A feasible trajectory $\psi_{t}=\{x(t), x(t)\}_{t=0}^{\infty}$, $(x(0), x(0)) \in \Delta$, is called synchronised trajectory. A feasible trajectory starting from $(x(0), y(0)) \in D-\Delta$, that is, with $x(0) \neq y(0)$, is synchronised if $|x(t)-y(t)| \rightarrow 0$ as $t \rightarrow+\infty$.

With regards to the dynamics of synchronised trajectories, we first recall the result proved in Proposition 1.

An attractor $A$ located on the invariant set $\Delta$ exists for $T_{b}$ if products are not too complementary ( $d$ is not too low) and the market share bonus $b$ is not too large (see Figures 3(a) and 3(c)). This fact can also be confirmed by considering the following properties of map $\phi(x): \phi(0)=0$, $\lim _{x \rightarrow 1^{-}} \phi(x)=-\infty, \phi^{\prime}(0)>1 \forall b \in(0, \bar{b}), \phi^{\prime \prime}(x)<0 \forall x \in$ $[0,1)$. As a consequence, there exists a unique $x_{-} \in(0,1)$ such that $\phi\left(x_{-}\right)=0$ and a unique maximum point $x_{M} \in\left(0, x_{-}\right)$ such that $\phi\left(x_{M}\right)$ is the maximum value of $\phi$ in $[0,1)$. This implies that $\phi$ is unimodal in $\left[0, x_{-}\right]$. By considering that $\phi\left(x_{M}\right)$ increases when $d$ decreases and $\phi\left(x_{M}\right) \rightarrow+\infty$ if $d \rightarrow$ -1 , then a $\bar{d}$ does exist such that $\phi\left(x_{M}\right)>x_{-}$if $-1<d<\bar{d}$ (see Figure 3(b)). On the other hand, by considering the role of parameter $b$, a cascade of period doubling bifurcations is observed when $b$ decreases (see Figure 3(c)). 

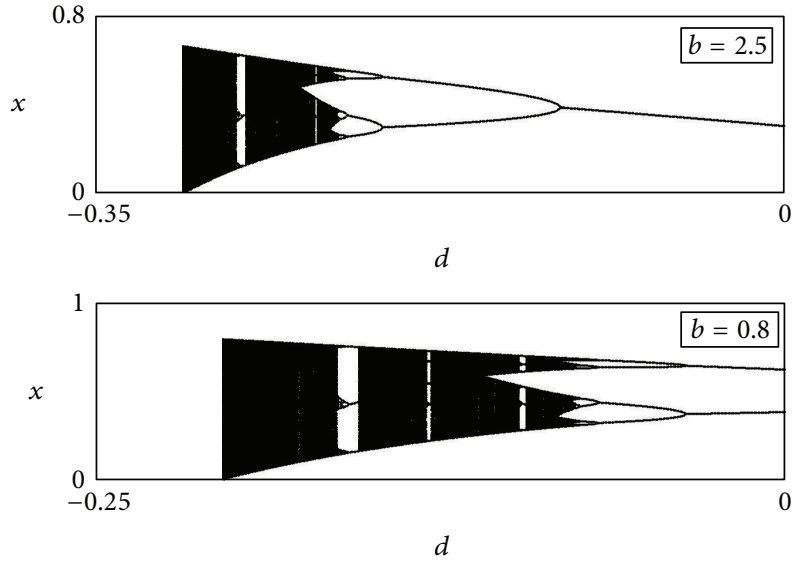

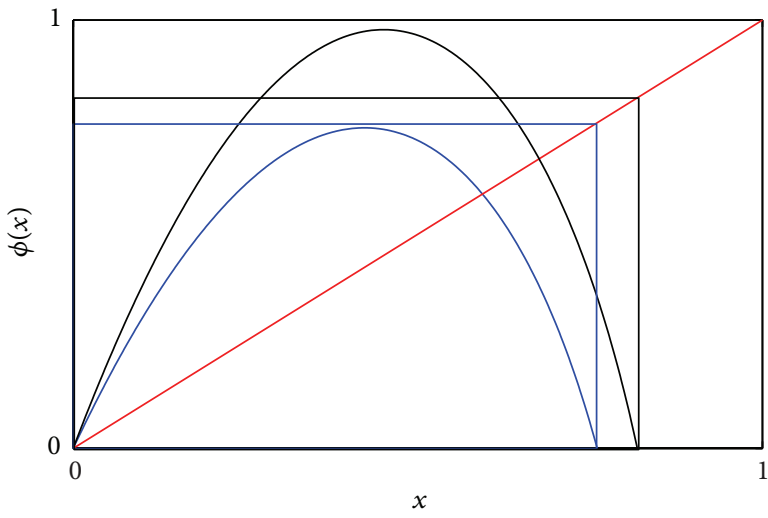

$d=-0.2$
$-\Delta$
$-d=-0.4$

(a)

(b)
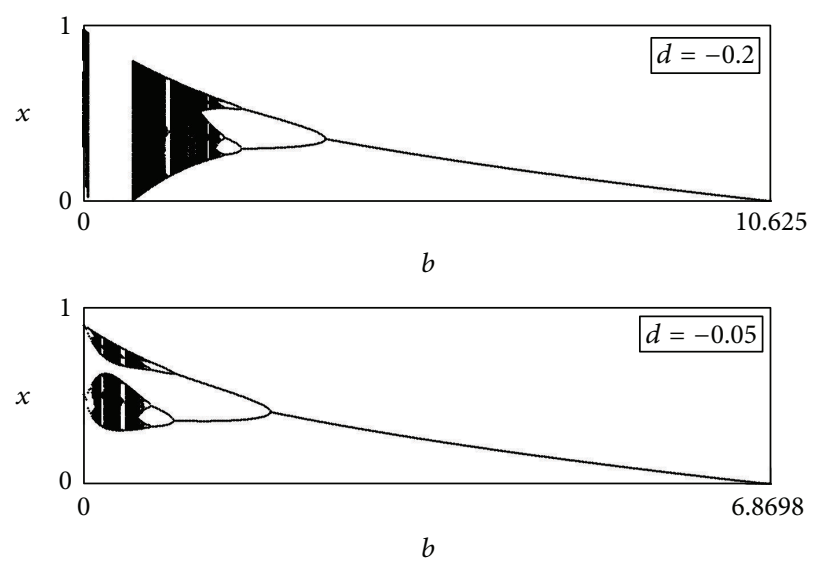

(c)

Figure 3: Parameter values: $w=0.5, \alpha=1.5$. (a) One-dimensional bifurcation diagrams with respect to $d$ for two fixed $b$ values. (b) Map $\phi$ is plotted for different $d$ values and $b=1$. (c) One-dimensional bifurcation diagrams with respect to $b(0<b<\bar{b})$ for two fixed $d$ values.

By taking into account the previous results and looking at the one-dimensional bifurcation diagrams in Figures 3(a) and 3(c), it can be observed that synchronised trajectories converge to the unique interior fixed point if $b$ is close to $\bar{b}$ (the manager bonus is close to its upper limit). In addition, similar to what occurs with the logistic map, cycles can emerge due to period doubling bifurcation of $\phi$ when $d$ decreases (i.e., the degree of complementarity between products increases). This evidence is also confirmed when products are substitutes, thus proving that complexity in synchronised trajectories arises when moving away from the hypothesis of independent products.

Let us consider now a duopoly with identical players that start from different feasible initial conditions and let $A \subseteq \Delta$ be an attracting set of $\phi$. If $A=E_{b}^{*}$ for $d=0$, then by considering (13) and Proposition 3 the following proposition holds.

Proposition 6. Let $x_{b}^{*}$ be an attracting fixed point of $\phi$ for $d=$ 0 . Then, $E_{b}^{*}$ is an attracting fixed point of $T_{b}$ and $\exists d_{-}<0$ such that $E_{b}^{*}$ is an attracting fixed point of $T_{b} \forall d \in\left(d_{-}, 0\right) \cap(-1,0)$. At $d=d_{-}$, the fixed point $E_{b}^{*}$ loses stability along the diagonal.

Proof. Let $d=0$ and assume that $x_{b}^{*}$ is an attracting fixed point of $\phi$; that is, $\phi^{\prime}\left(x_{b}^{*}\right)=\lambda_{b \|}\left(E_{b}^{*}\right) \in(-1,1)$. Since $\lambda_{b \|}\left(E_{b}^{*}\right)=$ $\lambda_{b \perp}\left(E_{b}^{*}\right)$, then $E_{b}^{*}$ is an attracting fixed point of $T_{b}$. As both $\lambda_{b \|}\left(E_{b}^{*}\right)$ and $\lambda_{b \perp}\left(E_{b}^{*}\right)$ are continuous with respect to $x_{b}^{*}$ and $d$, then $\exists I_{-}(0)$ such that $\left|\lambda_{b \|}\left(E_{b}^{*}\right)\right|<1$ and $\left|\lambda_{b \perp}\left(E_{b}^{*}\right)\right|<1$ $\forall d \in\left(d_{-}, 0\right) \cap(-1,0)$, and $E_{b}^{*}$ is an attracting fixed point of $T_{b}$. Finally, since $\lambda_{b \|}\left(E_{b}^{*}\right)<\lambda_{b \perp}\left(E_{b}^{*}\right) \forall d \in(-1,0)$ and they are both increasing with respect to $d$, then as $d$ crosses $d_{-}$the eigenvalue $\lambda_{b \|}\left(E_{b}^{*}\right)$ must cross -1 ; that is, $E_{b}^{*}$ loses its stability along the diagonal.

According to Proposition 6, if synchronised trajectories converge to $x_{b}^{*}$ for $d=0$, then trajectories starting from feasible initial conditions close to it, with $x(0) \neq y(0)$, are synchronised in the long term as long as $d \in I_{-}(0)$. If $d$ decreases further, the fixed point loses stability firstly 
along the diagonal. This contrasts with the result obtained in Fanti et al. [13] where products are substitutes. In fact, if $d$ passes from zero to positive values, the fixed point first loses transverse stability and consequently the trajectories are not synchronised. Therefore, synchronisation in this model is strictly related to the assumption of complementarity between products.

If $A$ consists of a $m$-cycle, then, similarly to what happens for the fixed point, several numerical computations show that if $A$ is a $m$-cycle for $d=0$, then the $m$-cycle loses stability firstly along the diagonal when $d$ decreases, so that synchronisation may occur. Different from the case of substitutability, this evidence confirms that when products are complements, players may coordinate their behaviour towards a situation in which prices are equal (and the market is equally shared). In our analysis it is also stressed that the emergence of synchronisation with negative values of $d$ also confirms the result obtained in Fanti et al. [18] with profitmaximising firms.

Consider now a more complex situation; that is, $A$ is a chaotic attractor on $\Delta$. In order to study its transverse stability, it is possible to use the procedure proposed in Bischi et al. [14], Bischi and Gardini [19], and Bignami and Agliari [20]. Recall that the transverse Lyapunov exponent is defined as follows:

$$
\Lambda_{b \perp}=\lim _{N \rightarrow \infty} \frac{1}{N} \sum_{n=0}^{N} \ln \left|\lambda_{b \perp}\left(x_{n}\right)\right|,
$$

where $x_{0} \in A$ and $x_{n}$ is a generic trajectory generated by $\phi$.

If $x_{0}$ belongs to a generic aperiodic trajectory embedded within the chaotic set $A$, then $\Lambda_{b \perp}$ is the natural transverse Lyapunov exponent $\Lambda_{b \perp}^{n}$, where natural indicates that the exponent is computed for a typical trajectory taken in the chaotic attractor $A$. Since infinitely many cycles (all unstable along the diagonal) are embedded inside the chaotic attractor $A$, a spectrum of transverse Lyapunov exponents can be defined and the natural transverse Lyapunov exponent represents a sort of weighted balance between the transversely repelling and transversely attracting cycles. If all cycles embedded in $A$ are transversely stable, that is $\Lambda_{b \perp}^{\max }<0$, then $A$ is asymptotically stable in the Lyapunov sense for the twodimensional map $T_{b}$. Nevertheless, it may occur that some cycles embedded in the chaotic set $A$ become transversely unstable; that is, $\Lambda_{b \perp}^{\max }>0$, while $\Lambda_{b \perp}^{n}<0$. In such a case, $A$ is not stable in the Lyapunov sense but it is a stable attractor in the Milnor sense. If a Milnor attractor of $T_{b}$ exists, then some transversely repelling trajectories can be embedded in a chaotic set which is attracting only on average. In addition, such transversely repelling trajectories can be reinjected toward $\Delta$ so that their behaviour is characterised by some bursts far from the diagonal, before synchronization or convergence towards a different attractor. This situation is called on-off intermittency.

In order to investigate the existence of a Milnor attractor $A$, we numerically estimate the natural transverse Lyapunov exponent $\Lambda_{b \perp}^{n}$, represented with respect to $b$ in Figure 4(a) for a fixed negative value of $d$. It is possible to observe that it can take negative values. As an example, we consider $b=2.07$ at which $\Lambda_{b \perp}^{n}<0$ while $\Lambda_{b \perp}^{\max }>0$ and the one-dimensional map $\phi$ exhibits a 4-piece chaotic attractor. $A$ is an attractor of system $T_{b}$ belonging to the diagonal (see Figure 4(b)), but a trajectory starting from an initial condition that does not belong to the diagonal has a long transient before converging to $A$ (see Figure 4(c)). In fact, by considering the difference $x(t)-y(t)$ for any $t$ we can observe that the transient part of the trajectory is characterised by several bursts away from $\Delta$. The typical on-off intermittency phenomenon occurs. The whole trajectory starting from $x(0)=0.1$ and $y(0)=0.2$ is shown in Figure 4(d).

The study of the geometrical properties of the critical lines may be used to estimate the maximum amplitude of the bursts by obtaining the boundary of a compact trapping region of the phase plane in which the on-off intermittency phenomena are confined. Following Mira et al. [21], we obtain the boundary of the absorbing area in Figure 4(e) for the case presented in Figure 4(d). Observe that such a region contains the whole trajectory presented in Figure 4(d). However, not all trajectories are synchronised as $T_{b}$ also admits a coexisting attractor, that is, a 2-period cycle whose basin is depicted in orange in Figure 4(f).

If $T_{b}$ admits an attractor $A \subset \Delta$ and there exist feasible trajectories starting from interior points that are not synchronised, then the question of multistability has to be considered. In fact, several attractors may coexist (each of which with its own basin of attraction) so that the selected long-term state becomes path dependent, as in the situation shown in Figure 4(f). In this case, the structure of the basins of different attractors becomes crucial to predict the longterm outcome of the economic system. In Figure 5(a), the unique Nash equilibrium is locally stable, as the market share bonus is close to its upper limit. It is also globally stable, in the sense that it attracts all feasible trajectories taken into the interior of the feasible set $D$ (that represents economic meaningful initial conditions).

If we compute $\partial \bar{b} / \partial d$, then it is possible to observe that $\bar{b}$ increases as $d$ decreases, and $\bar{b} \rightarrow+\infty$ as $d \rightarrow-1^{+}$. As a consequence, in order to obtain the convergence to the unique Nash equilibrium, $b$ must be set at higher levels as the degree of horizontal product differentiation $d$ decreases (i.e., products tend to be more complementary). Furthermore, if products are complements, then $\lambda_{b \|}$ crosses -1 as $b$ decreases and a flip bifurcation occurs along the invariant set $\Delta$; that is, a 2-period cycle appears close to the fixed point and it is stable along the diagonal. By taking into account the result proved in Proposition 3, when this bifurcation occurs, $\lambda_{b \perp}$ is still smaller than 1 in modulus and consequently (immediately after the first flip bifurcation) the 2-period cycle attracts all interior feasible trajectories. However, if $b$ is still decreased, a period doubling bifurcation cascade occurs along the diagonal, so that more complex bounded attractors (such as periodic cycles) may exist on $\Delta$ around the unstable Nash equilibrium. As a consequence, the long-term synchronised dynamics may be characterised by bounded periodic (or even aperiodic) oscillations around the Nash equilibrium. If the attractor $A$ is transversely unstable, the situation may become very complicated, as it is shown in Figure 5(b) where a 2-period cycle attracts all synchronised trajectories, while two attractors coexist out of the diagonal: a 2-period cycle 


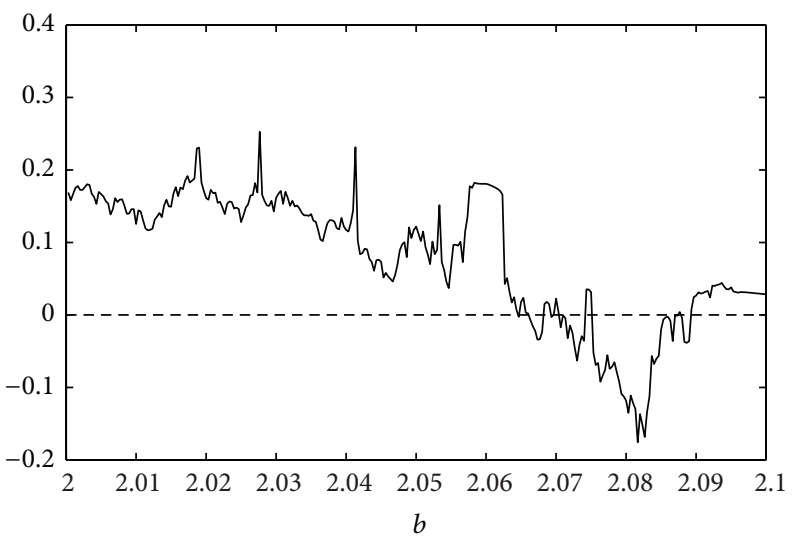

(a)

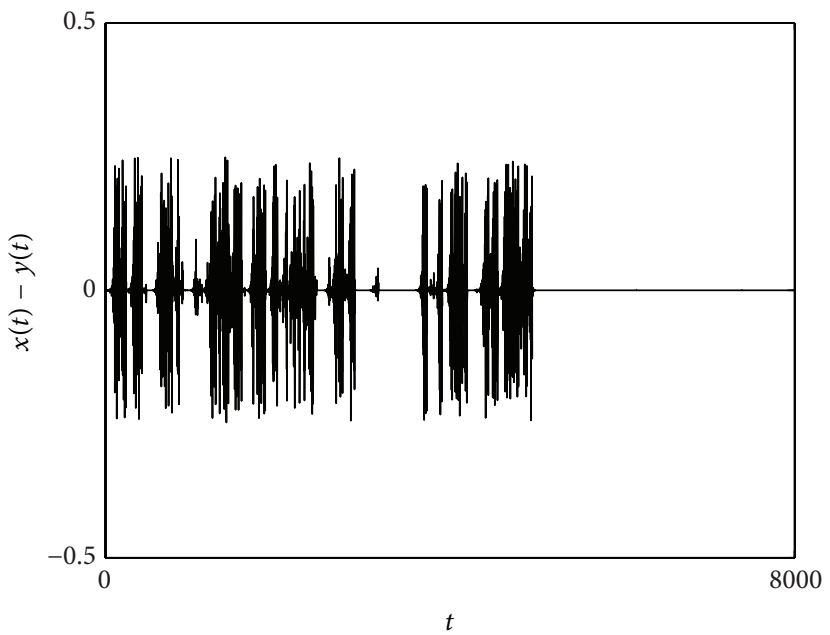

(c)

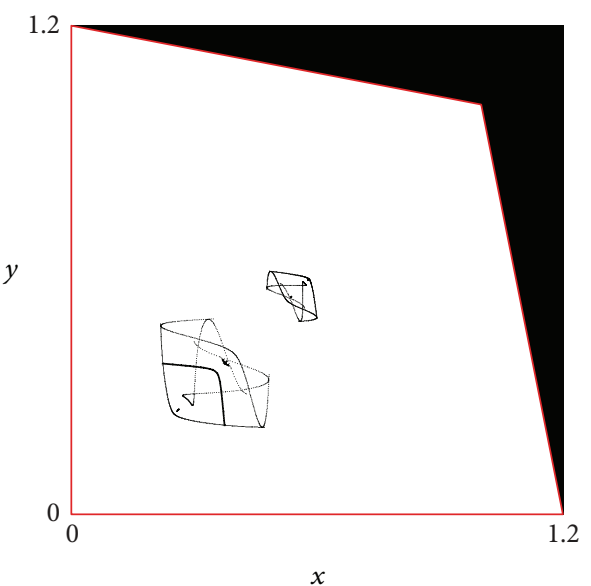

(e)

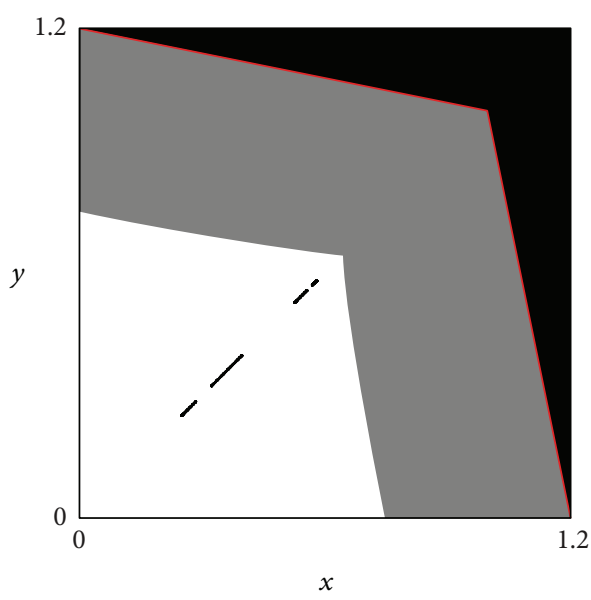

(b)

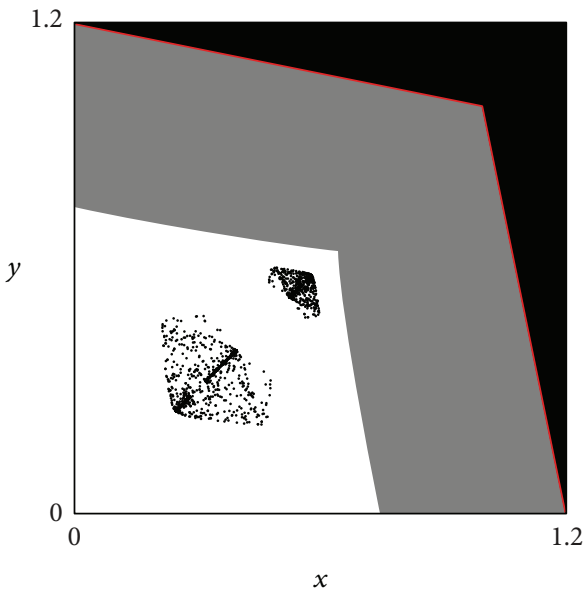

(d)

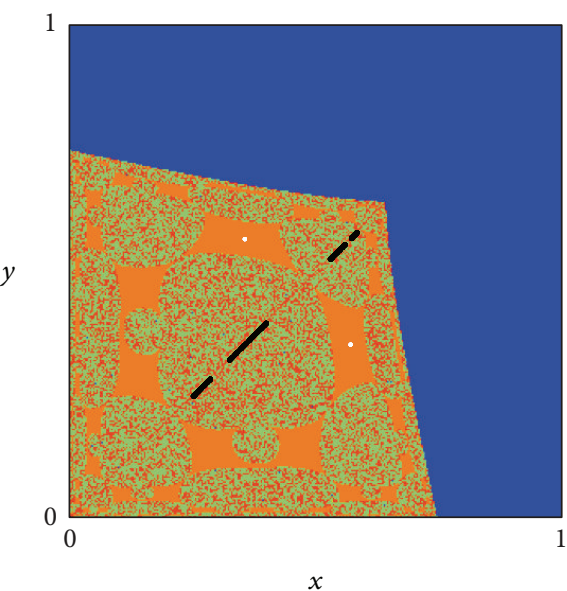

(f)

Figure 4: Parameter values: $\alpha=1.5, d=-0.2$, and $w=0.5$. (a) The natural transverse Lyapunov exponent with respect to parameter $b$. (b) Four-piece chaotic attractor $A$ of system $T_{b}$ belonging to the diagonal for $b=2.07$. (c) Bursts away from the diagonal before synchronization for $b=2.07, x(0)=0.1$, and $y(0)=0.2$. (d) The whole trajectory starting from initial condition as in (c) and converging to the attractor in (b). (e) The minimal absorbing area in which on-off intermittency phenomenon occurs for the same parameters as in (c). (f) The attractor $A$ coexists with an attracting 2-period cycle for $b=2.07$. 


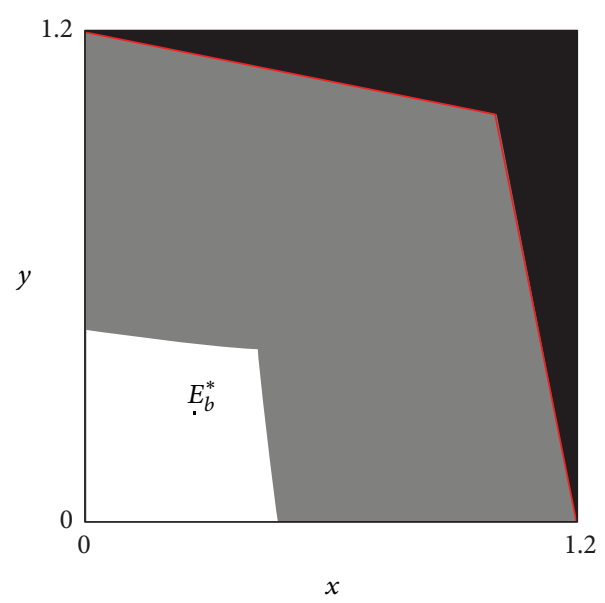

(a)

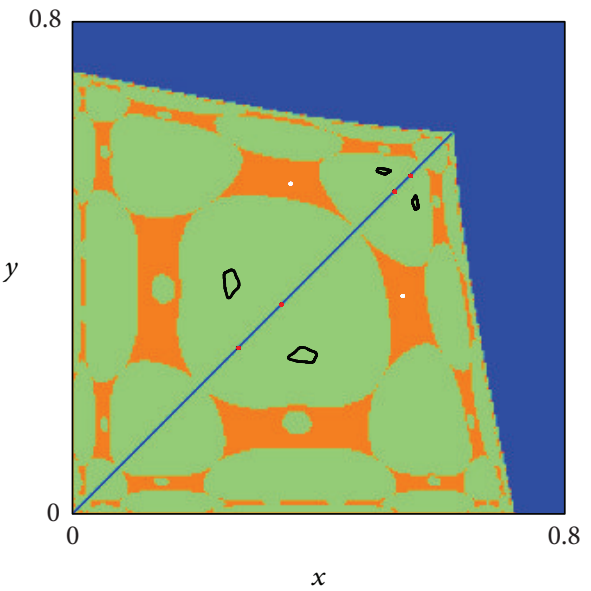

(c)

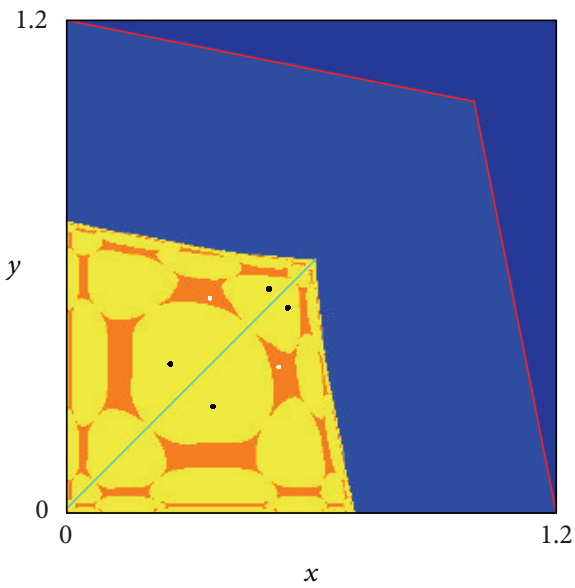

(b)



(d)

Figure 5: Parameter values: $\alpha=1.5$ and $w=0.5$. (a) The white region represents the basin of $E_{b}^{*}$ for $b=5.1$ and $d=-0.2$. (b) A 4-period cycle together with a 2-period cycle coexists with the attractor $A$ belonging to the diagonal (a two-period cycle) for $b=2.4$ and $d=-0.2$. (c) For $b=2.3$, a 4 -piece quasi-periodic attractor has been created, $d=-0.2$. (d) A particular scenario is presented for $b=0.2$ in the case of independent products $d=0$.

whose basin is depicted in orange and a 4-period cycle whose basin is depicted in yellow; note that the periodic points are in symmetric position with respect to the diagonal. As the parameter $b$ decreases, a further flip bifurcation occurs and a 4-period cycle is created on the diagonal (see Figure 5(c)); furthermore, the 4-period cycle existing out of the diagonal becomes a stable focus and then undergoes a Neimark-Sacker bifurcation at which it becomes a 4-cyclic attractor formed by a 4-piece quasi-periodic attractor (green basin) coexisting with a 2-period cycle (orange basin), while synchronisation is avoided. This is how the situation presented in Figure 4(f) is approached: a final bifurcation that causes the transition to more complex basin boundaries occurs. Consequently to the final state sensitivity it is impossible to predict the long-term outcomes of the economy.

Finally, we consider the case in which products are independent from each other and each manager behaves as a monopolist $(d=0)$. When a flip bifurcation along the diagonal creates a $k$-period cycle, it can be observed that a $k$-period cycle is simultaneously created out of the diagonal as the eigenvalues of cycles embedded into the diagonal are identical. As a consequence, any period doubling bifurcation along $\Delta$ is associated with a period doubling bifurcation orthogonal to $\Delta$. A similar phenomenon of multistability is presented in Bischi and Kopel [22]. This case is shown in Figure 5(d): the green points represent initial conditions converging to a 4-period cycle on the diagonal while the yellow points represent initial conditions converging to a 4-period cycle out of the diagonal. This scenario occurs when the 2-period cycle along the diagonal undergoes the second period doubling bifurcation: two stable 4-period cycles are created, one along the diagonal (red points) and one with periodic points symmetric to it (black points); these two stable cycles coexist with the 2-period cycle previously created out of the diagonal (white points). Observe that an economy starting far away from the diagonal may become synchronised, as the basin of attraction $A$ on the diagonal is comprised of several nonconnected sets. This result is 

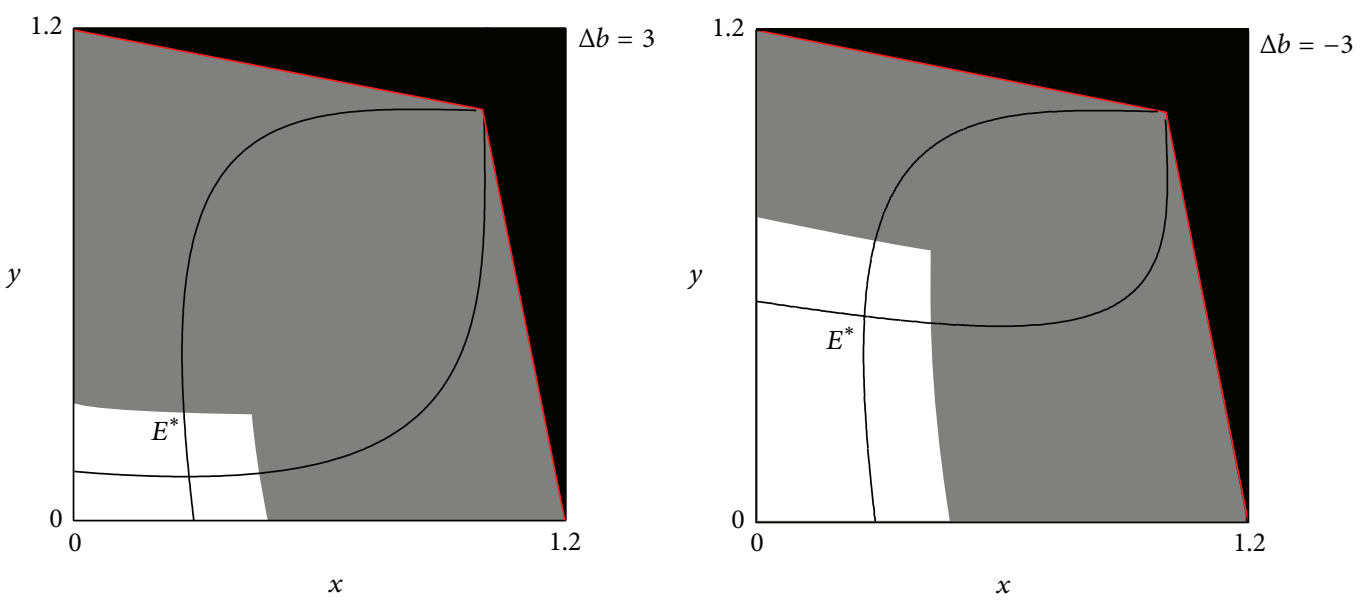

(a)



(b)

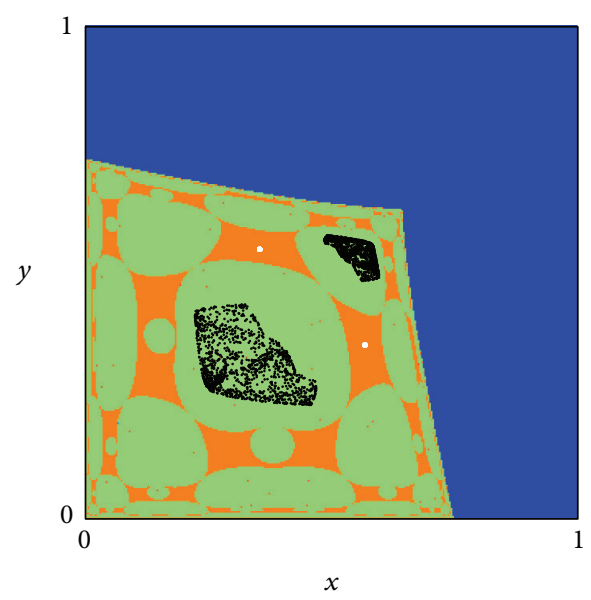

(c)

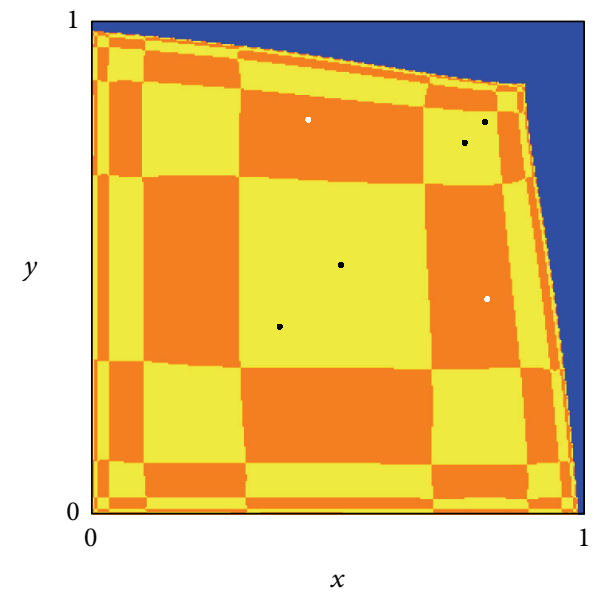

(d)

Figure 6: (a) Parameter values: $\alpha=1.5, w=0.5, b=5.1$, and $d=-0.2$. The Nash equilibrium for positive and negative values of $\Delta b$. (b) If $\Delta b=-0.3$, the Nash equilibrium is unstable and a 2-period cycle is globally stable. (c) A complex attractor $A$ (black points) coexists with a 2-period cycle (white points) if $\alpha=1.5, w=0.5, b=2.07, d=-0.2$, and $\Delta b=0.15$. (d) Coexisting attractors if $b=0.2, d=0$ while $\Delta b=0.01$. 
relevant from an economic point of view. In fact, it implies coordination even though the manager hired in each firm behaves as a monopolist in his own market.

5.1. The Asymmetric Case. We now consider the case in which managers' bonuses are evaluated differently; that is, $b_{1} \neq b_{2}$. Obviously, in this case $\Delta$ is no longer invariant (i.e., if the firms start from the same initial feasible condition $(x(0), x(0)) \in$ $D$, they will behave differently in the long term), so that synchronisation cannot occur. However, similar to the case in which products are substitutes, multistability still emerges. Assume $b_{1}=b$ and $b_{2}=b+\Delta b$, where $\Delta b \in(-b,+\infty)$.

With regard to the existence of the Nash equilibrium, we recall that Proposition 1 states a necessary condition; that is, parameter $d$ should not be too close to its extreme value -1 and, in addition, $b$ and $\Delta b$ should not be too high. A Nash equilibrium, if it exists, is given by a point $\left(x^{*}, y^{*}\right) \in Q$ such that $F\left(x^{*}, y^{*}\right)=G\left(x^{*}, y^{*}\right)=0$. As a consequence, an interior fixed point can be obtained by considering the intersection points of the two curves $F(x, y)=0$ and $G(x, y)=0$ in the phase plane. Of course, if these curves intersect in a point $E^{*}=\left(x^{*}, y^{*}\right) \in Q$, then it is a Nash equilibrium for $T$.

By considering the analytical properties of $F$ and $G$, numerical simulations allow us to conclude that the main results of Fanti et al. [13] are confirmed also when products are complements or independent. These results are collected in the following list.

(i) If the Nash equilibrium exists, then it is unique such that the equilibrium price is higher for the variety associated with a lower market share bonus (see Figure 6(a)).

(ii) If the Nash equilibrium is locally stable in the symmetric case, then it is also locally stable in the asymmetric case if and only if the perturbation on $b$ is small enough (i.e., $\Delta b$ is close to zero).

(iii) In the case of heterogeneity, the Nash equilibrium loses stability via a flip bifurcation at which it becomes a saddle point and a stable 2-period cycle appears close to $E^{*}$ (see Figure 6(b)).

(iv) Synchronised trajectories do not emerge and synchronisation cannot occur, while multistability still emerges (compare Figures 6(c) to 4(f)).

To better describe point (iv) above, we recall that a situation in which synchronisation may occur is depicted in Figure 4(f): the symmetric system $T_{b}$ admits a complex attractor on the diagonal that coexists with a 2-period cycle out of the diagonal. If we consider a slight difference between weights attached to market share bonuses, that is, $\Delta b$ is small enough, we obtain the situation depicted in Figure 6(c): the attractor on the diagonal disappears while a complex attractor coexists with the 2-period cycle previously found.

Similarly, observe that, with independent products and homogeneous managers, three coexisting attractors are owned (see Figure 5(d)). This situation drastically changes if $\Delta b=0.01$. In fact, as shown in Figure 6(d), a small perturbation on $b$ causes the disappearance of the attracting 4-period cycle symmetric to the diagonal, while a 4-period cycle close to the diagonal persists together with the 2-period cycle existing out of the diagonal. Obviously, due to the heterogeneity between the weights $b_{i}$, the shape of the boundaries of the coexisting attractors is no longer symmetric with respect to the diagonal.

Although the managers' behaviours are no longer coordinated, it is interesting to stress that, different from the case of substitutability between products, the structure of the basin of attraction seems to become simpler than under homogeneous delegation contracts.

\section{Conclusions}

This paper has studied the mathematical properties of a nonlinear duopoly game with price competition and market share delegation contracts. The main aim was to extend the analysis carried out by Fanti et al. [13] to the case in which products are complementary or independent. The most important result is that the interaction between the degree of complementarity and the delegation variable (which affects managerial bonuses) may produce synchronisation in the long term. This result does not emerge when products are substitutes.

From an economic point of view, synchronisation is relevant because it implies coordination between players. Then, in a model with managerial firms and market share delegation contracts, coordination can (resp., cannot) hold when products are complements (resp., substitutes). In addition, we have also shown that multiple attractors may exist so that initial conditions matter.

\section{Conflict of Interests}

The authors declare that there is no conflict of interests regarding the publication of this paper.

\section{References}

[1] W. J. Baumol, "On the theory of oligopoly," Economica, vol. 25, no. 99, pp. 187-198, 1958.

[2] E. F. Fama and M. C. Jensen, "Separation of ownership and control," Journal of Law and Economics, vol. 26, no. 2, pp. 301$325,1983$.

[3] J. Vickers, "Delegation and the theory of the firm," The Economic Journal, vol. 95, pp. 138-147, 1985.

[4] C. Fershtman and K. Judd, "Equilibrium incentives in oligopoly," The American Economic Review, vol. 77, pp. 927-940, 1987.

[5] C. Fershtman, "Managerial incentives as a strategic variable in duopolistic environment," International Journal of Industrial Organization, vol. 3, no. 2, pp. 245-253, 1985.

[6] N. H. Miller and A. I. Pazgal, "The equivalence of price and quantity competition with delegation," RAND Journal of Economics, vol. 32, no. 2, pp. 284-301, 2001.

[7] N. H. Miller and A. I. Pazgal, "Relative performance as a strategic commitment mechanism," Managerial and Decision Economics, vol. 23, no. 2, pp. 51-68, 2002.

[8] T. Jansen, A. van Lier, and A. van Witteloostuijn, "A note on strategic delegation: the market share case," International Journal of Industrial Organization, vol. 25, no. 3, pp. 531-539, 2007. 
[9] T. Jansen, A. V. Lier, and A. van Witteloostuijn, “On the impact of managerial bonus systems on firm profit and market competition: the cases of pure profit, sales, market share and relative profits compared," Managerial and Decision Economics, vol. 30, no. 3, pp. 141-153, 2009.

[10] M. Kopel and L. Lambertini, "On price competition with market share delegation contracts," Managerial and Decision Economics, vol. 34, no. 1, pp. 40-43, 2013.

[11] S. Gray, "Cultural perspectives on the measurement of corporate success," European Management Journal, vol. 13, no. 3, pp. 269275, 1995.

[12] S. C. Borkowski, "International managerial performance evaluation: a five country comparison," Journal of International Business Studies, vol. 30, no. 3, pp. 533-555, 1999.

[13] L. Fanti, L. Gori, C. Mammana, and E. Michetti, "Local and global dynamics in a duopoly with price competition and market share delegation," Chaos, Solitons \& Fractals, vol. 69, pp. 253-270, 2014.

[14] G.-I. Bischi, L. Stefanini, and L. Gardini, "Synchronization, intermittency and critical curves in a duopoly game," Mathematics and Computers in Simulation, vol. 44, no. 6, pp. 559-585, 1998.

[15] G. I. Bischi, L. Gardini, and M. Kopel, "Analysis of global bifurcations in a market share attraction model," Journal of Economic Dynamics and Control, vol. 24, no. 5-7, pp. 855-879, 2000.

[16] G.-I. Bischi and F. Lamantia, "Nonlinear duopoly games with positive cost externalities due to spillover effects," Chaos, Solitons and Fractals, vol. 18, pp. 805-822, 2002.

[17] S. Brianzoni, C. Mammana, and E. Michetti, "Local and global dynamics in a discrete time growth model with nonconcave production function," Discrete Dynamics in Nature and Society, vol. 2012, Article ID 536570, 22 pages, 2012.

[18] L. Fanti, L. Gori, C. Mammana, and E. Michetti, “The dynamics of a Bertrand duopoly with differentiated products: synchronization, intermittency and global dynamics," Chaos, Solitons \& Fractals, vol. 52, pp. 73-86, 2013.

[19] G.-I. Bischi and L. Gardini, "Global properties of symmetric competition models with riddling and blowout phenomena," Discrete Dynamics in Nature and Society, vol. 5, no. 3, pp. 149$160,2000$.

[20] F. Bignami and A. Agliari, "Synchronization and on-off intermittency phenomena in a market model with complementary goods and adaptive expectations," Studies in Nonlinear Dynamics \& Econometrics, vol. 14, no. 2, pp. 1-15, 2010.

[21] C. Mira, L. Gardini, A. Barugola, and J. C. Cathala, Chaotic Dynamics in Two-Dimensional Non-Invertible Maps, World Scientific, Singapore, 1996.

[22] G. I. Bischi and M. Kopel, "Multistability and path dependence in a dynamic brand competition model," Chaos, Solitons and Fractals, vol. 18, no. 3, pp. 561-576, 2003. 


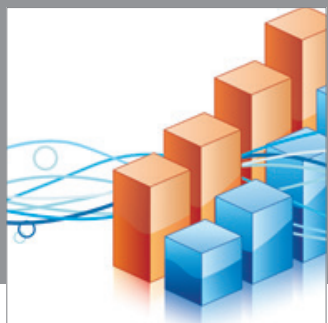

Advances in

Operations Research

mansans

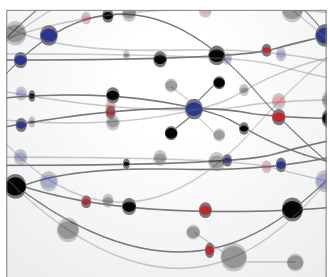

The Scientific World Journal
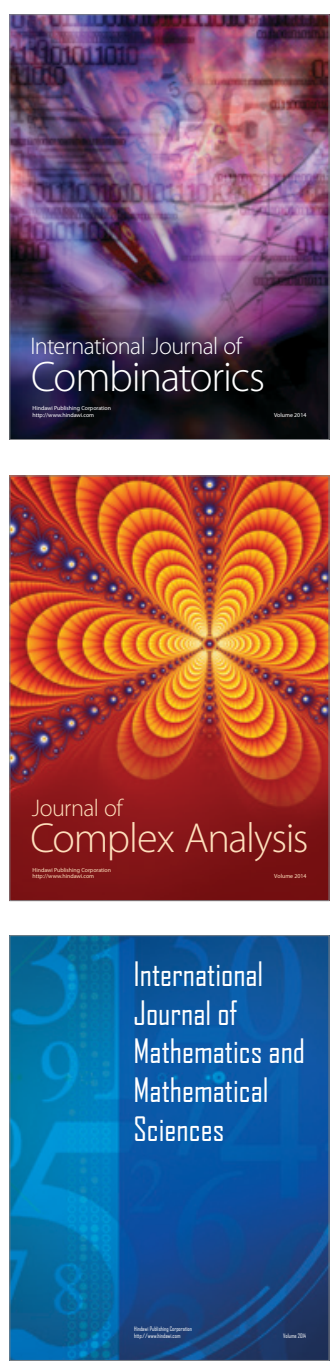
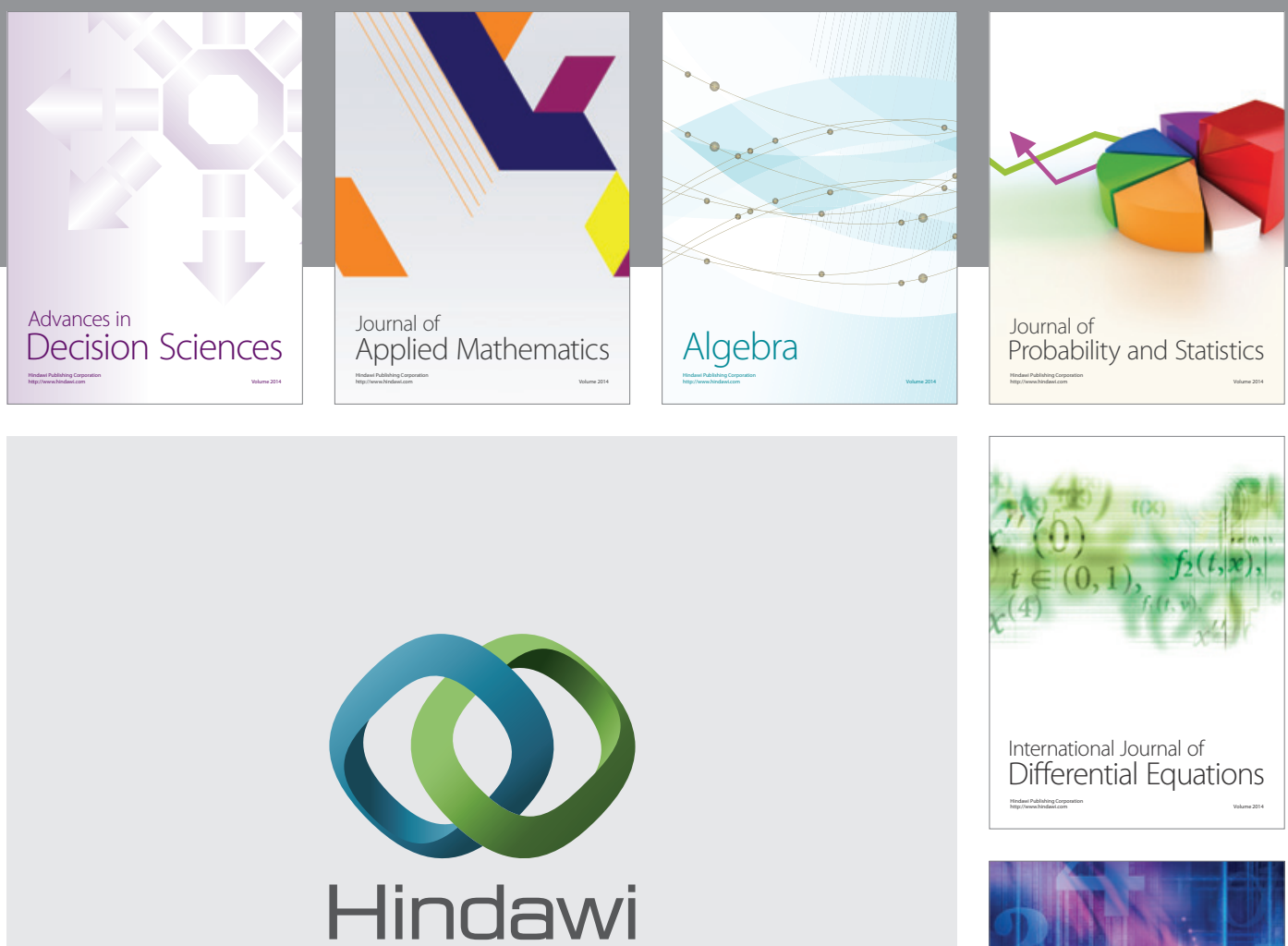

Submit your manuscripts at http://www.hindawi.com
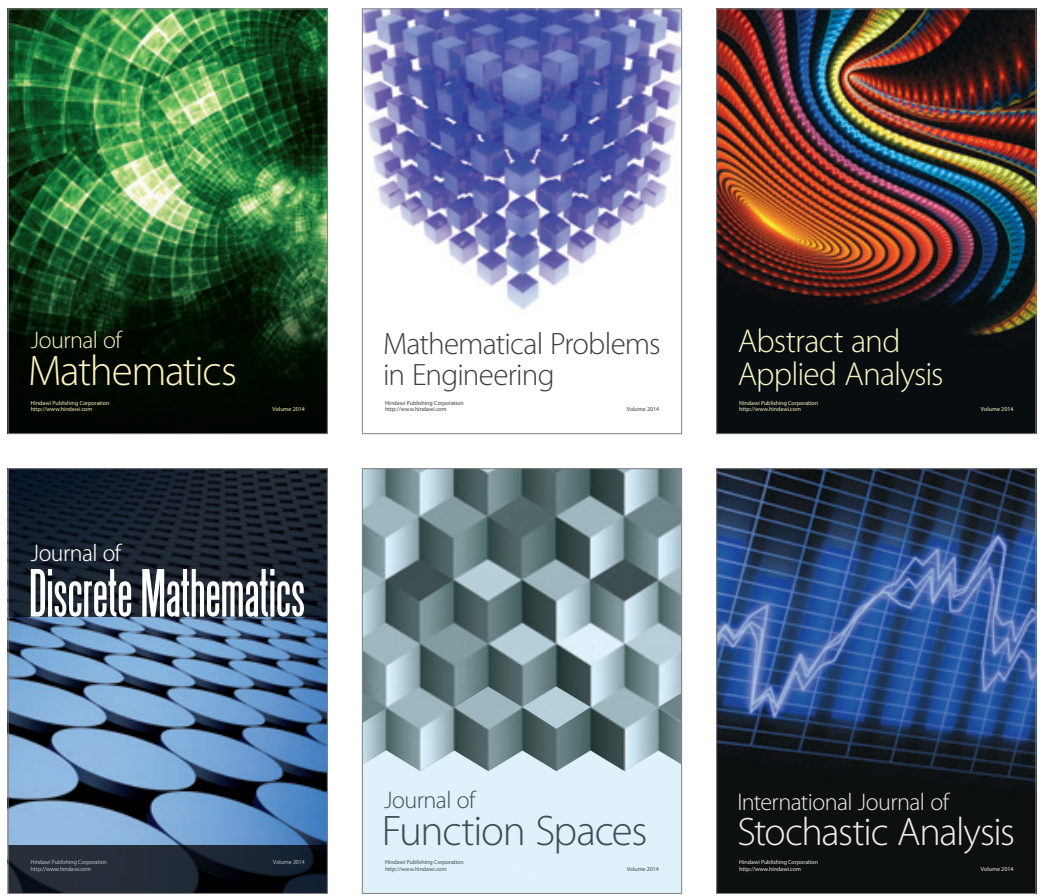

Journal of

Function Spaces

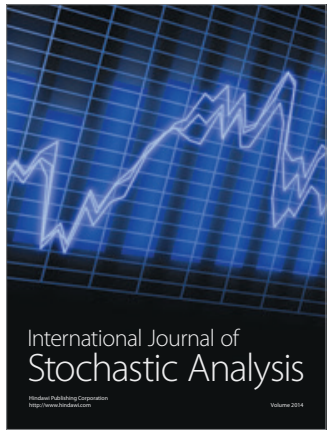

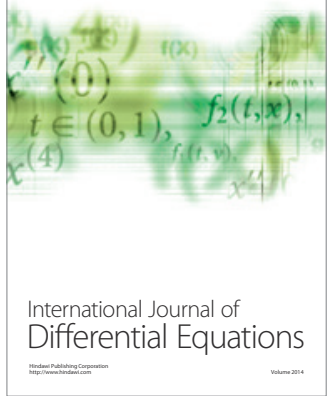
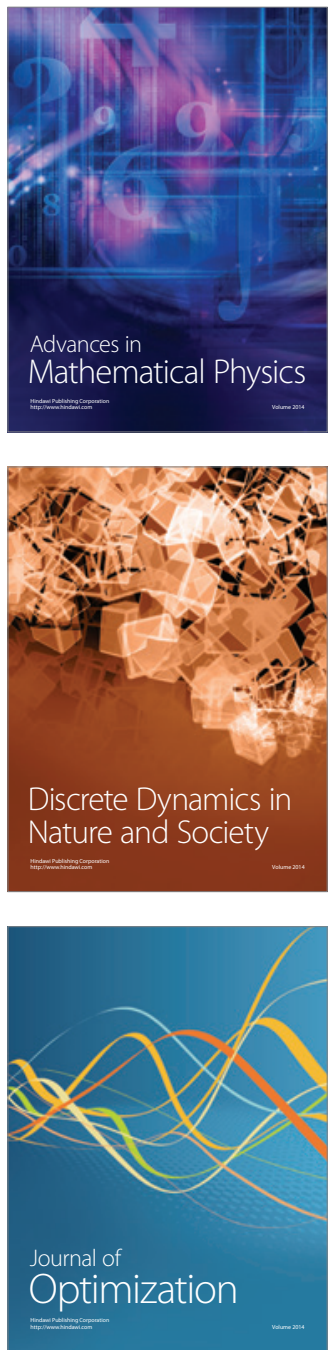